\title{
Efficacy of duloxetine in achieving response and remission Michael Bauer*
}

Address: Department of Psychiatry and Psychotherapy, Charité University Medicine Berlin, Berlin, Germany

* Corresponding author

from International Society on Brain and Behaviour: 2nd International Congress on Brain and Behaviour

Thessaloniki, Greece. 17-20 November 2005

Published: 28 February 2006

Annals of General Psychiatry 2006, 5(Suppl I):S2I doi:I0.I I86/I744-859X-5-SI-S2 I

Deficiencies in the activity of specific pathways for serotonin (5-HT, 5-hydroxytryptamine), and norepinephrine (NE) have long been hypothesized to account for the symptoms of major depressive disorder (MDD). More than 30 years ago, it was noted that pharmacological agents that enhance the availability of the monoamine neurotransmitters serotonin, norepinephrine, and dopamine, produced a clinically meaningful improvement in mood in patients suffering from MDD. This clinical observation led to the development of the so-called 'monoamine hypothesis' of depression, which proposed that functional disruptions in these neurotransmitter systems mediate the behavioural and physiological symptoms (e.g. painful physical symptoms) of depression and related conditions. The pharmacotherapy of MDD has relied principally on the development of agents that alter or increase the functional activity of these classical neurotransmitter systems [1].

A major disadvantage of the older drugs, such as the classic tricyclic antidepressants (TCAs), is their safety profile. Adverse events resulting from the use of such drugs are frequently reported by patients as a reason for stopping treatment. Newer agents, acting primarily only on one neurotransmitter system, such as the selective serotonin reuptake inhibitors (SSRI) are generally accepted to be effective antidepressants with a favourable side-effect profile relative to many of the older drugs. However, only approximately $30 \%$ of patients may be expected to achieve remission of illness with SSRI monotherapy. Recent clinical evidence has accumulated that the selective enhancement of both 5-HT and NE systems provide a more robust clinical effect, e.g., in terms of higher remission rates [1].

Duloxetine hydrochloride is a novel, balanced and potent dual reuptake inhibitor of both 5-HT and NE. Duloxet- ine's efficacy in the treatment of major depression has been established in a series of placebo-and comparatorcontrolled trials, and offers consistent efficacy on both the emotional and painful physical symptoms of depression, together with good tolerability and ease of use [2]. Data indicate that duloxetine is an important addition to the existing treatment options for MDD because it treats the complete depressive condition, thus allowing more patients to not only attain, but also sustain, a state of remission - the ultimate goal of acute depression treatment.

\section{References}

I. Bauer M, Whybrow PC, Angst J, Versiani M, Möller HJ, Task Force on Treatment Guidelines for Unipolar Depressive Disorders: World Federation of Societies of Biological Psychiatry (WFSBP) Guidelines for biological treatment of unipolar depressive disorders, Part I: Acute and continuation treatment of major depressive disorder. World J Biol Psychiatr 2002, 3:5-43.

2. Nemeroff CB, Schatzberg AF, Goldstein DJ, Detke MJ, Mallinckrodt, Lu Y, Tran PV: Duloxetine for the treatment of major depressive disorder. Psychopharmacol Bull 2002, 36:106-132. 